\title{
Biodegradation testing: so many tests but very little new innovation
}

\author{
Ji-Dong Gu* \\ School of Biological Sciences, The University of Hong Kong, Pokfulam Road, Hong Kong SAR, People’s Republic of \\ China
}

\begin{abstract}
Biodegradation tests have been conducted for testing the degradation of environmental pollutants for at least half a century, but little innovations and new information have been made available from the more recent efforts. In this context, substrate, microorganisms, enrichment and degradation are discussed critically to summarize the essential approaches for biodegradation testing and also the new directions in this area of research. The information intends to provide some essential assistance to individuals working on this topic in their research and applications.
\end{abstract}

Keywords: biodegradation, intermediates, biochemical pathway, enrichment, culture medium, bioremediation

*Correspondence to: Ji-Dong Gu, School of Biological Sciences, The University of Hong Kong, Pokfulam Road, Hong Kong SAR, People’s Republic of China; Email: jdgu@hku.hk

Received: November 26, 2015; Accepted: February 19, 2016; Published Online: March 9, 2016

Citation: Gu J-D, 2016, Biodegradation testing: so many tests but very little new innovation. Applied Environmental Biotechnology, vol.1(1): 92-95. http://dx.doi.org/10.26789/AEB.2016.01.007.

\section{Introduction}

$\mathrm{B}$ iodegradation is crucial to ecosystem function for nutrient cycling and it also plays a central role in understanding the fate and toxicity of environmental pollutants in the anthropocene. The concept of biodegradation delineated by Martin Alexander has contributed to the training of several generations of scientists and graduate students to work on this subject of organic persistent chemicals throughout their careers ${ }^{[1]}$. Recently, research on biodegradation has been shifted more and more to the developing countries, especially the emerging economies such as China, India, Brazil, etc., due to the economic development ${ }^{[2,3]}$, but many recent reported studies have failed to show new innovative understanding and novel knowledge beyond what had been established decades ago. Because of this, it is not only important but also necessary to reiterate the significance of essential knowledge and the necessity of innovation on degradation tests and research to advance our new knowledge to the next high level for advancement of science.

\section{Biodegradation and its Misunderstandings}

Biodegradation is defined as the breakdown of organic chemicals, including pollutants, to innocuous products such as $\mathrm{CO}_{2}, \mathrm{CH}_{4}$ and $\mathrm{H}_{2} \mathrm{O}$ by the action of (micro)organisms including archaea, bacteria and fungi. The transformation process from a selective chemical to mineralization products involves a series of intermediate biochemical steps to achieve the degradation, but intermediate products may be more toxic than the initial parent compounds in some cases, so chemical modification of parent compounds is not always a preferred strategy in pollutant management and remediation. Based on this information, degradation should focus on mineralization of the chemical to completion if possible from the environmental point of views so that bioremediation as a goal can be achieved in laboratory settings, and more importantly in practice, to deal with polluted sites. There are at least several misunderstandings in setting up degrada-

Biodegradation testing: so many tests but very little new innovation. (C) 2016 Ji-Dong Gu. This is an Open Access article distributed under the terms of the Creative Commons Attribution-NonCommercial 4.0 International License (http://creativecommons.org/licenses/by- nc/4.0/), permitting all non-commercial use, distribution, and reproduction in any medium, provided the original work is properly cited. 
tion experiments and gathering research data in laboratory research; incorrect information and practices are widely reported in literature. Beginners need to fully understand these and plan effectively and meaningfully to achieve specific research objectives and advance science.

Firstly, the target pollutant under investigation is not the sole source of carbon and energy for microorganisms in the investigations because complex cultural medium containing some or all of the following: glucose, yeast extract, and/or nutrient broth are used to enhance the growth of the microbial populations. Under such conditions, the increase of microbial population is not the result of the direct metabolic utilization of the target pollutant solely and the activity of the microorganisms involved. Such results on degradation are misleading to people entering to this research field without prior knowledge or in-depth background information because co-metabolism is the biochemical process involved $^{[1,4]}$. Therefore, the first rule in the practice is to use defined mineral medium with known chemical composition to achieve the goal of degradation by microorganisms capable of utilizing the test chemical as the sole source of carbon and energy ${ }^{[5-11]}$. Given the fact that microbial degradation can be achieved using the established defined medium through rigorous effort, biodegradation pathway can be further constructed through identification of some intermediates for better understanding for the sequences of biochemical steps in the degradation.

Secondly, another key component in the biodegradation investigation is to enrich a population of consortium or isolate a pure culture of strain so that degradation experiments can be carried out with known microorganism or a stable population of microorganisms in consortium. To achieve this, enrichment should be implemented and this procedure is often overlooked in many studies. In the enrichment process, the source of inoculum is also very important, and some knowledge about the site and physical and chemical characteristics are essential. Many studies simply use this step briefly as a safe security for passing the information check than seriously put any energy and effort to maximize the value of this process. In my previous experience to enrich a 3-methylindole (skatole)-degradation methanogenic consortium, municipal wastewater from the city of Roanoke, Virginia yielded a consortium transforming 3-meyhlindole to 3-methyloxindole and the biochemical reaction stopped there without any further transforma- tion $^{[6]}$. A parallel study involving degradation tests for atrazine, cyanazine, dicamaba and paraquat using wetland sediment from East Piedmont region of Virginia allowed me to test the degradation of 3-methylindoel using a difference source of inoculum and the results showed the mineralization of the 3-methylindole could be achieved with this new source of inoculum containing the microorganisms ${ }^{[7]}$. Time and efforts spent on the inoculum may pay off in the end if one can spend more time on them meaningfully and consistently.

Thirdly, the dynamics of both microbial population and substrate concentration should be monitored and shown together in the results presented. It is common and often the case that no microbial population information is presented in many investigations, but decline of substrate concentration is shown as the single evidence of degradation, not even the control data without inoculation. In this case, changes in concentrations of microorganisms and the substrate/pollutant should be shown simultaneously to allow the evaluation of possible degradation of the pollutant by the implicated microorganisms over the time of incubation. In addition, to confirm the degradation by microorganisms, an abiotic control is a must to be set up in parallel to the biologically active treatments so that chemical stability can be evaluated without biases from chemical transformation, e.g., hydrolysis as an example ${ }^{[9,10]}$. Another control should be included, but rarely used, is the killed or sterilized inoculum to show any effects from denatured proteins of the inoculum cells. With full consideration of these, the experimental set up can provide strong evidence on the degradability of the pollutants by the pure culture or consortium of microorganisms.

\section{Improving Biodegradation Research Practice}

In today's scientific research laboratories equipped with new analytical and molecular instruments available, more can be done to achieve better confirmation on biodegradation of pollutants and also the microorganisms involved. In terms of chemical analysis, both gas chromatography (GC) and high-performance liquid chromatography (HPLC) are common instruments available and GC-Mass Spectrometer (MS) and HPLCMS are also becoming routine. More advanced analytical instruments with double MS are also used for chemical identification and quantification. The analytical capability in research has been improved greatly in the last 20 years and it is a normal practice 
to quantify and identify the degradation products/intermediates for a comprehensive knowledge about the degradation processes and capability of the microorganisms. Similarly, molecular techniques on manipulation of genes involved have also become an essential practice in the laboratory to PCR amplify the target gene, quantify such gene abundance and monitor gene expression. High throughput sequencing techniques are readily used by both biologists and non-biologists to reveal the information of microbial community for much more detailed information of community composition of microorganisms. Both transcriptomics and proteomics are used to further obtain the active metabolic processes and also the proteins expressed ${ }^{[12]}$. In addition, stable isotope probing allow a direct linkage between the substrate and the microorganisms utilizing it in pure culture or environmental conditions, which enhances our research capability greatly for better understanding the in situ population and the metabolically active microorganisms without taking them out of the natural niche ${ }^{[13]}$.

Environmental pollutants are mostly hydrophobic and have very low solubility in water. As a result, they do not dissolve very well in water and culture medium, limiting the access of them as sources of carbon and energy to microorganisms. A reasonably high portion of results reported involves such chemicals at concentrations far above their physical solubility limit and degradation of them has been presented. Such results deserve more caution for interpretation even if solvent was used to facilitate the dissolution of the chemicals because the hydrophobicity of the pollutants will not make them favorable for assimilation and then metabolism. Since the soluble concentrations used are too low, the so-called degradation observed could be biased or simply due to the loss from volatilization, adsorption of the chemical to culture flasks and also onto the cellular biomass.

These described above are the basis required when testing the concept of biodegradation in its framework to advance our understanding of the metabolic processes and the microorganisms involved for the target chemicals so that bioremediation can be proceeded in in situ condition to achieve cleanup. Without thorough background knowledge, planning and experimental design, the results obtained cannot support the objectives of a study and such data have unfortunately already penetrated into the published literature. Because of these, young investigators need to be equipped with basic essential knowledge to make their right decision critically and selectively to contribute to the scientific knowledge through the paradigm shift framework based on solid scientific data. With better knowledge and in-depth understanding, technological development can be fostered to tackle the real world problems through research based on solid foundation $^{[4]}$.

\section{Conclusion}

Science is a process involving constant efforts from many people and different cultures over generations to establish some sound basis of a specific area of research and knowledge. If the information generated was not up to the standard of the scientific test over time, they will be proven incorrect, but can also lead to the wrong direction of the efforts by others to follow. Paradigm shift of scientific concept is largely based on accumulation of scientific information proven correct to advance the science ${ }^{[14]}$. Without such a building process, science can hardly make any meaningful progress. Due to these explained as above, I wish to use this commentary to show a case through my experiences in teaching, research and serving as editor and reviewers, so that young scientists, especially graduate students, can benefit from them in their research to avoid repeating the incorrect practice and take the wrong results as true.

\section{Conflict of Interest and Funding}

There is no conflict of interest. Research in my laboratory has been supported by funding from Hong Kong SAR Government and RGC GRF grants.

\section{References}

1. Alexander M, 1999, Biodegradation and Bioremediation, $2^{\text {nd }}$ edn. Academic Press, San Diego.

2. Gu J-D, 2014, Assessment of ecosystem health and ecotoxicology through chemical analysis and modeling. Ecotoxicology, vol.23(4): 475-479. http://dx.doi.org/10.1007/s10646-014-1206-x.

3. Gu J-D and Wang Y, 2013, A new era for geomicrobial ecotoxicology in environmental science research. International Biodeterioration \& Biodegradation, vol.76: $1-2$.

http://dx.doi.org/10.1016/j.ibiod.2012.06.024.

4. Mitchell R and Gu J-D, 2010, Environmental Microbiology, $2^{\text {nd }}$ edn. John Wiley, New York.

5. Gu J-D, 2003, Microbiological deterioration and degradation of synthetic polymeric materials: recent research advances. International Biodeterioration \& Biodegra- 
dation, vol.52(2): 69-91.

http://dx.doi.org/10.1016/S0964-8305(02)00177-4.

6. Gu J-D and Berry D F, 1991, Degradation of substituted indoles by an indole-degrading methanogenic consortium. Applied and Environmental Microbiology, vol.57(9): 2622-2627.

7. Gu J-D and Berry D F, 1992, Metabolism of 3-methylindole by a methanogenic consortium. Applied and Environmental Microbiology, vol.58(8): 2667-2669.

8. Gu J-D and Pan L, 2006, Comparison of growth characteristics of three bacteria involved in degrading rubber. Journal of Polymers and the Environment, vol.14(3): 273-279. http://dx.doi.org/10.1007/s10924-006-0016-5.

9. Gu J-D and Wang Y, 2013, Microbial transformation of phthalate esters: diversity of hydrolytic esterases, in Environmental Contamination - Health Risks, Bioavailability and Bioremediation, Wong M H (ed.). CRC Press, Boca Raton: 313-346. http://dx.doi.org/10.1201/b12531-19.

10. Li J and Gu J-D, 2006, Biochemical cooperation between Klebsiella oxytoca Sc and Methylobacterium me- sophilium $\mathrm{Sr}$ for complete degradation of dimethyl isophthalate. The Interactions Between Sediments and Water: 205-210.

http://dx.doi.org/10.1007/978-1-4020-5478-5_21.

11. Yip K C-W and Gu J-D, 2016, A novel bacterium involved in the degradation of 2-methylindole isolated from sediment of Inner Deep Bay of Hong Kong. Applied Environmental Biotechnology, vol.1(1): 52-63. http://dx.doi.org/10.18063/AEB.2006.01.008.

12. Jiang X W, Wang J, Chan L L, et al. 2015, Comparison of three protein extraction procedures from toxic and non-toxic dinoflagellates for proteomics analysis. Ecotoxicology, vol.24(6): 1395-1406.

http://dx.doi.org/10.1007/s10646-015-1514-9.

13. Mbadinga S M, Wang L Y, Zhou L, et al. 2011, Microbial communities involved in anaerobic degradation of alkanes. International Biodeterioration \& Biodegradation, vol.65(1): 1-13.

http://dx.doi.org/10.1016/j.ibiod.2010.11.009.

14. Kuhn T S, 1970, The Structure of Scientific Revolutions, $2^{\text {nd }}$ edn (enlarged). University of Chicago Press, Chicago, IL. 